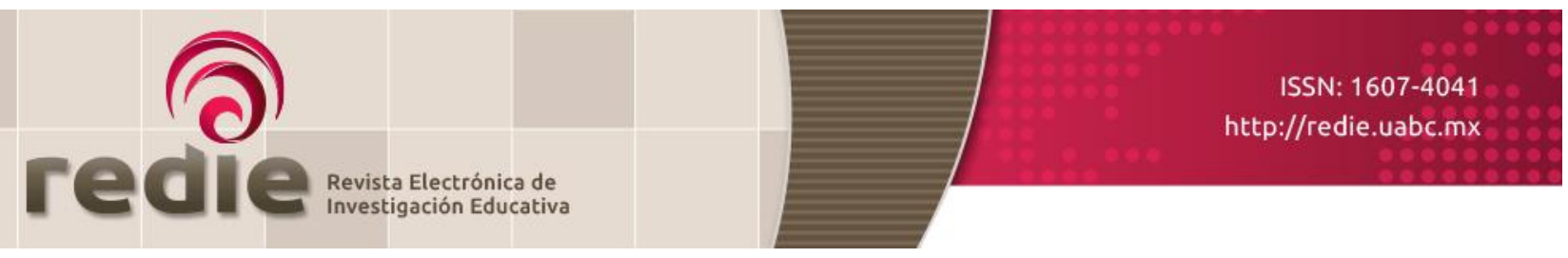

Vol. 19, Núm. 4, 2017

\title{
Condición de becario y rendimiento académico en estudiantes de una universidad peruana
}

\section{Scholarship Holders and Academic Performance at a Peruvian University}

\author{
Diana Alicia Salinas Oviedo (*) diana.salinas@upn.edu.pe \\ Ariadna Elizabeth Hernández (*) ariadna.hernandez@upn.edu.pe \\ Miguel Barboza-Palomino (*) mbarbozapalomino@outlook.com.pe
}

(1) Universidad Privada del Norte

(Recibido: 7 de abril de 2016; Aceptado para su publicación: 9 de julio de 2016)

Cómo citar: Salinas, D. A., Hernández, A. E. y Barboza-Palomino, M. (2017). Condición de becario y rendimiento académico en estudiantes de una universidad peruana. Revista Electrónica de Investigación Educativa, 19(4), 124-133.

https://doi.org/10.24320/redie.2017.19.4.1348

\section{Resumen}

La investigación buscó analizar el efecto diferencial del programa Beca 18 promovido por el gobierno peruano en el rendimiento académico de los beneficiarios y no beneficiarios durante los estudios de una carrera universitaria. Para ello se ejecutó un estudio empírico cuantitativo de tipo ex post facto, analizando la relación de la condición de becario y su influencia en el rendimiento académico (promedio de calificaciones) en una muestra de 1,262 estudiantes (becarios=204; no becarios=1,058) de 9 programas académicos de la Universidad Privada del Norte (Perú). Los resultados indican que un alto porcentaje de los becados (99.5\%) logra aprobar el semestre académico; además, se constata que estos estudiantes alcanzan promedios de calificaciones superiores a los estudiantes no becarios. Se concluye que el programa Beca 18 tiene un efecto positivo en su rendimiento académico.

Palabras clave: Rendimiento académico, Educación Universitaria, Beca de estudios, Perú.

\section{Abstract}

This study sought to analyze the differential effect of the "Beca 18" program, promoted by the Peruvian government, on academic performance in beneficiaries and non-beneficiaries in university degree programs. An empirical, quantitative and ex post facto study was carried out to analyze the relationship between students' status as scholarship holders and the impact this had on academic performance (average grade) in a sample of 1,262 students (scholarship holders=204; non-scholarship holders=1,058) from 9 study programs at the Universidad Privada del Norte in Peru. The results show that a high percentage of scholarship holders (99.5\%) pass their academic semester. Furthermore, it was found that these students achieve higher grades than non-scholarship holders. It is concluded that the Beca 18 program has a positive effect on academic performance. 
Keywords: Academic performance, Universities, Scholarships, Peru.

\section{Introducción}

Como parte de las políticas de equidad e inclusión promovidas por el Estado peruano, en el año 2012 se implementó el programa Beca 18 -con el propósito de promover igualdad de oportunidades en el acceso a la educación superior a través de subvenciones educativas no reembolsables (El Comercio, 2012). El programa en mención no fue la primera iniciativa en política de becas; sin embargo, lo que caracteriza la propuesta es que está focalizada en la población de adolescentes y jóvenes (de 16 a 22 años) provenientes de estratos socioeconómicos de pobreza y extrema pobreza (Bolívar, 2005), a quienes se provee una dotación económica que contempla pensiones de estudios, materiales educativos, movilidad, alimentación, vivienda, seguro médico y gastos de titulación, además de incluir el servicio de acompañamiento y tutoría (Navarro, 2015).

La propuesta valora el componente del rendimiento académico, lo cual se visualiza en los mecanismos de selección y renovación (es otorgada a estudiantes que exhiben un buen desempeño académico en la etapa escolar) y su continuación está condicionada a la aprobación del semestre de estudios en la universidad, buscando garantizar la permanencia durante el período de formación profesional (Ministerio de Educación, 2014). Cabe destacar la estructura dual (apoyo económico y acompañamiento tutorial) del programa Beca 18, que a juicio de Cuellar (2011) convierte a un programa de beca en una propuesta educativa innovadora de inclusión.

En relación al rendimiento académico, Edel (2003) plantea que puede ser asumido como un indicador que expresa el resultado de un proceso de enseñanza-aprendizaje, factible de ser medido considerando los resultados de evaluación de cada asignatura, no obstante, resulta una variable compleja en cuya construcción participan una multiplicidad de factores. En el ámbito de la educación superior, diversos autores (Garbanzo, 2013; Garzón, Rojas, del Riesgo, Pinzón y Salamanca, 2010; Ruiz, Ruiz y Odstrcil, 2007) reafirman su naturaleza multicausal, señalando que en su configuración intervienen aspectos personales (capacidades cognitivas, hábitos de estudio, conocimientos previos, nivel de pensamiento formal, autoestima, motivaciones, habilidades sociales, inteligencia emocional, entre otros), los relacionados con el entorno familiar y social del estudiante (comunicación, dinámica y clima familiar; violencia, desempleo, inseguridad), los dependientes de la institución (material didáctico, equipamiento, planes de estudios actualizados) y los que dependen de los docentes (metodología de enseñanza, capacitación, reflexión y actualización de sus prácticas, uso de nuevas tecnologías).

Considerando la multiplicidad de factores que intervienen en la configuración del rendimiento académico se puede afirmar que adquieren un matiz distinto en grupos de estudiantes con desventajas económicas; sin embargo, existe evidencia que permite sustentar en términos generales que el desempeño académico está relacionado con las habilidades y aptitudes del estudiante, siendo la inteligencia la variable más estudiada y el componente más estable a la hora de predecirlo, tal como lo reportan Miñano, Cantero y Castejón (2008). Otra línea explicativa relaciona el rendimiento académico con factores motivacionales (González, Donolo, Rinaudo y Paoloni, 2011; Guitérrez y López, 2012). Por otra parte, en las últimas décadas se evidenció el nexo entre el desempeño académico y factores contextuales, que de acuerdo a Chiroleu (2009) implica poner atención en que los programas de subvenciones o becas no suprimen las ventajas o desventajas de origen de los estudiantes, las cuales suelen repercutir en su rendimiento académico.

Partiendo de esto y situando los criterios de selección y renovación del programa Beca 18, los beneficiarios exhibirían un rendimiento académico destacable; sin embargo, factores motivacionales y contextuales podrían perjudicar su desempeño, por ello se examinan resultados de estudios previos que abordaron la condición de becario y las implicancias en el rendimiento académico de los beneficiarios.

En el escenario peruano se encuentra Navarro (2015), quien comparó el rendimiento académico de estudiantes becarios y no becarios del programa Beca 18 de la Universidad Continental, para lo cual ejecutó un estudio descriptivo con los 25 estudiantes mejor posicionados en el ranking académico universitario al concluir un semestre de estudio, analizó los promedios de calificaciones, identificando que 
del total, el 68\% poseía la condición de becario. El estudio concluye que las actividades extracurriculares y de acompañamiento tutorial explican el éxito académico de los becarios.

De igual forma, Rivera (2012) sistematizó las experiencias de beneficiarios y ejecutores de programas de becas integrales para continuar estudios universitarios en la Pontificia Universidad Católica del Perú, para ello desarrolló un estudio cualitativo ejecutando talleres participativos con 30 estudiantes beneficiarios y 6 personas de la oficina de becas. Los beneficiarios identifican que un factor de éxito en relación al rendimiento académico lo constituyen las actividades de acompañamiento; por otro lado, los ejecutores señalan que se debe incidir en actividades informativas acerca de la utilidad de las becas para lograr la adecuada inclusión de los becarios en la universidad.

En el ámbito internacional, Catalán y Santelices (2015) abordaron las diferencias en el rendimiento académico de estudiantes becados y no becados que accedieron a una carrera universitaria en la Pontificia Universidad Católica de Chile en el período de 2007 a 2010. Se realizó un estudio descriptivo e inferencial para establecer las diferencias en relación al rendimiento académico con una muestra de 3,518 estudiantes becados y 27,574 estudiantes no becados. Los resultados indican que los beneficiarios de los programas de becas obtienen resultados similares en relación a los estudiantes no becados

Por otro lado, Gómez (2014) estudió el impacto de las becas escolares en el rendimiento académico de estudiantes de la Universidad Autónoma de Nuevo León (México), para lo cual trabajó con una muestra de 260 estudiantes que un semestre académico anterior no hubieran recibido beca, comparando el promedio de calificación obtenido en el semestre que ostentó la condición de becario con el ciclo académico en el cual no fue becario. Los resultados indican que el estatus de becado no impacta significativamente en el rendimiento académico de la muestra estudiada.

Por su parte, Martínez y Martínez (2013) plantearon como objetivo analizar las diferencias existentes entre el rendimiento académico de estudiantes beneficiarios y no beneficiarios de becas que cursan estudios de bachillerato en una institución educativa mexicana del estado de Oaxaca. Desarrollaron un estudio descriptivo que implicó la comparación de promedios de calificación de 246 estudiantes, encontrando que no existen diferencias estadísticamente significativas con relación a los promedios de calificación entre becarios y no becarios; sin embargo, constatan descriptivamente un mayor promedio de calificación académica en estudiantes becados.

Berlanga, Figuera y Pons-Fanals (2013) valoraron la incidencia de la beca salario sobre el rendimiento académico en el primer año de estudios universitarios, ejecutando una investigación de carácter descriptivo-comparativo de tipo ex post facto, trabajando con 10,394 estudiantes, de los cuales 642 poseían la condición de becados. Los resultados muestran que los beneficiarios de becas exhiben una mayor motivación académica, lo que se ve reflejado en la matrícula en varias asignaturas; sin embargo, suelen exhibir en algunos casos un rendimiento académico deficiente, lo que podría ser explicado por la exigencia para el cumplimiento de estándares de excelencia académica como condición de renovación de beca.

De la misma forma, Mediavilla (2013) analizó el impacto de las becas sobre la posibilidad de finalizar con éxito el nivel secundario postobligatorio en España. Para lo cual desarrolló un estudio cuasi-experimental aplicando la estrategia Propensity Score Matching a partir de la Encuesta de Condiciones de Vida aplicada en el 2006. Los resultados indican la existencia de efectos diferenciales positivos en la finalización exitosa de sus estudios en el caso de las mujeres y estudiantes que provienen de hogares desfavorecidos.

Asimismo, Martínez (2012) estudió los efectos del programa de becas educativas "Oportunidades" sobre la reducción del ausentismo escolar, ejecutando un estudio econométrico, encuestando en 1,130 hogares a beneficiarios del programa y 900 hogares no beneficiarios pertenecientes a los estados mexicanos de Nuevo León, Tamaulipas y Coahuila. Los resultados muestran que el programa "Oportunidades" no genera impacto en la reducción del ausentismo escolar, y concluye que eso se debe a la existencia de un alto porcentaje en relación a la variable estudiada. 
Por último, Estavillo, Peralta y Torres (2011) compararon el impacto de la obtención de una beca en el rendimiento académico en estudiantes de la Universidad de la República (Uruguay) durante el período 2002 a 2004. Utilizaron la metodología de Propensity Score Matching, que permitió -a través de un vector observable de variables- estimar las diferencias en relación a su desempeño académico en estudiantes becados $(\mathrm{N}=905)$ y no becados $(\mathrm{N}=242)$. Los resultados evidencian que estudiantes becados exhiben un mayor avance en sus ciclos académicos y suelen ser exitosos académicamente al egresar.

Parte de las investigaciones expuestas reportan efectos positivos de la condición de becario en el rendimiento académico, destacando como variable explicativa la existencia de un acompañamiento tutorial; por otro lado, hay estudios que indican lo contrario; al constituir el programa "Beca 18" -la política pública más importante del Estado peruano en materia de inclusión educativa, que en la práctica provee una dotación económica y servicio de tutoría-, la investigación apuntó a conocer el impacto que genera en el desempeño académico de sus beneficiarios, el cual se comparó con el rendimiento académico de un grupo de estudiantes no beneficiarios, planteando el objetivo de analizar el efecto diferencial en relación al rendimiento académico en estudiantes beneficiarios y no beneficiarios del programa Beca 18 de la Universidad Privada del Norte (UPN).

\section{Método}

Se desarrolló un estudio empírico con metodología cuantitativa, de tipo ex post facto prospectivo con una variable independiente (Montero y León, 2007). La investigación se realizó en una muestra de 1,262 estudiantes de la UPN, de los cuales el $16 \%$ fueron becarios ( $N=204)$, y el $84 \%$ no becarios $(N=1058)$. Cabe mencionar que según el Ministerio de Educación del Perú [MINEDU] (2014) la UPN alberga 206 becarios que representa el 2.8\% del total nacional de beneficiarios al 2014.

La muestra seleccionada cumplió con los criterios de exclusión: 1) estudiantes de carreras en el que estudiaran becarios del programa; 2) estudiantes que estuvieran matriculados al menos en una materia en el semestre académico analizado. De igual forma, el estudio incluyó a estudiantes de ambos sexos y de las tres sedes de la universidad ubicadas en las ciudades de Lima, Cajamarca y Trujillo.

Tabla I. Composición de la muestra de estudiantes del segundo semestre (2014-II) de la upN por sede

\begin{tabular}{r|r|r|r|r|r|r}
\hline Sede & Becario & No becario & Total general & \% Becario & \% No becario & \% Total \\
\hline Cajamarca & 108 & 158 & 266 & 8.56 & 12.52 & 21.08 \\
Lima & 9 & 631 & 640 & 0.71 & 50.00 & 50.71 \\
Trujillo & 87 & 269 & 356 & 6.89 & 21.32 & 28.21 \\
Total general & 204 & 1058 & 1262 & 16.16 & 83.84 & 100.00 \\
\hline
\end{tabular}

\subsection{Procedimiento estadístico}

Se determinaron las variables del estudio, identificándose como variable independiente la condición de becario del programa Beca 18 y como variable dependiente el rendimiento académico. La condición de becario del programa Beca 18 operativamente implicó identificar si el estudiante formaba parte o no del programa de becas, definida como variable de tipo cualitativa dicotómica; mientras que el rendimiento académico operativamente definido como el promedio de calificación que obtienen los estudiantes en la trayectoria académica e identificada como variable de tipo cuantitativo continuo.

Dadas las características de las variables, se eligió el estadístico inferencial $t$ de Student para determinar las diferencias entre el rendimiento académico entre beneficiarios de Beca 18 y estudiantes sin beca. El contraste de las hipótesis estadísticas se realizó asumiendo un riesgo de error del $5 \%(a=0.05)$. Conforme a las exigencias de la prueba, se constataron los supuestos de distribución normal y homogeneidad de varianzas.

Para el supuesto de distribución normal se aplicó la prueba Kolmogorov-Smirnov, la cual arrojó una distribución asimétrica negativa (-.192), lo que implicó una transformación de datos obteniendo una 
distribución normal, como se esperaba. En la tabla Il se observan los valores de la muestra inicial (columna promedio) y los valores con los datos transformados (columna promedio normalizado).

Tabla II. Prueba de Kolmogorov-Smirnov para una muestra

\begin{tabular}{|c|c|c|c|}
\hline & & Promedio & $\begin{array}{c}\text { Promedio } \\
\text { normalizado }\end{array}$ \\
\hline & $\mathrm{N}$ & 1262 & 1261 \\
\hline \multirow{2}{*}{ Parámetros normales ${ }^{a, b}$} & Media & 12.3637 & 12.3790 \\
\hline & Desviación típica & 4.07843 & 4.00858 \\
\hline \multirow{3}{*}{ Diferencias más extremas } & Absoluta & .192 & .027 \\
\hline & Positiva & .103 & .027 \\
\hline & Negativa & -.192 & -.023 \\
\hline \multicolumn{2}{|c|}{ Z de Kolmogorov-Smirnov } & 6,823 & .952 \\
\hline \multicolumn{2}{|c|}{ Sig. asintót. (bilateral) } & .000 & .325 \\
\hline
\end{tabular}

En la constatación del supuesto de homocedasticidad de varianzas se aplicó la prueba de Levene. Esta prueba nos proporciona un valor $F(19.824)$ y un valor de significatividad ( $p$-valor $=0.00$ ) que indicaban que las varianzas eran distintas, contrario a lo que se esperaba.

Tabla III. Prueba Levene para igualdad de varianzas

\begin{tabular}{lr|c|c}
\hline & & F & Sig. \\
\hline Normal promedio & Se han asumido varianzas iguales & 19.824 & .000 \\
& No se han asumido varianzas iguales & ---- & --- \\
\hline
\end{tabular}

Al no cumplir con el supuesto de homogeneidad, para el contraste de medias se realizó a través del cálculo del valor $t$ para varianzas heterogéneas. Este procedimiento consistió en obtener un valor calculado de $t$ (TCalculado) para compararlo con el valor teórico de $t$ (Tcrítica) obtenido de la tabla de distribución teórica de tStudent. En el contraste, la regla de decisión implicó que si el valor de TCalculado fuera superior al valor de Tcrítica, se rechazaría la hipótesis nula y, por ende, se aceptaría las diferencias de medias.

Por último, se calculó el tamaño del efecto con el objetivo de constatar la magnitud práctica de la diferencia estadística en relación al rendimiento académico entre el grupo de becarios y no becarios, obteniéndose la $d$ de Cohen (Ledesma, Macbeth y Cortada, 2008), que implicó la diferencia de medias de los promedios de calificaciones de ambos grupos dividido por la desviación estándar.

\section{Resultados}

\subsection{Análisis descriptivo de las variables}

El análisis descriptivo de la muestra, tal como se presenta en la figura 1, permite observar que un alto porcentaje de la cohorte analizada (82.09\%) aprueba las materias de estudios (puntaje <12 puntos, en una escala vigesimal). No obstante, se puede observar que casi la totalidad de becarios (99.5\%) supera el mínimo de puntaje exigido para aprobar los cursos, mientras que este porcentaje es relativamente menor en los estudiantes sin beca (78.7\%). 


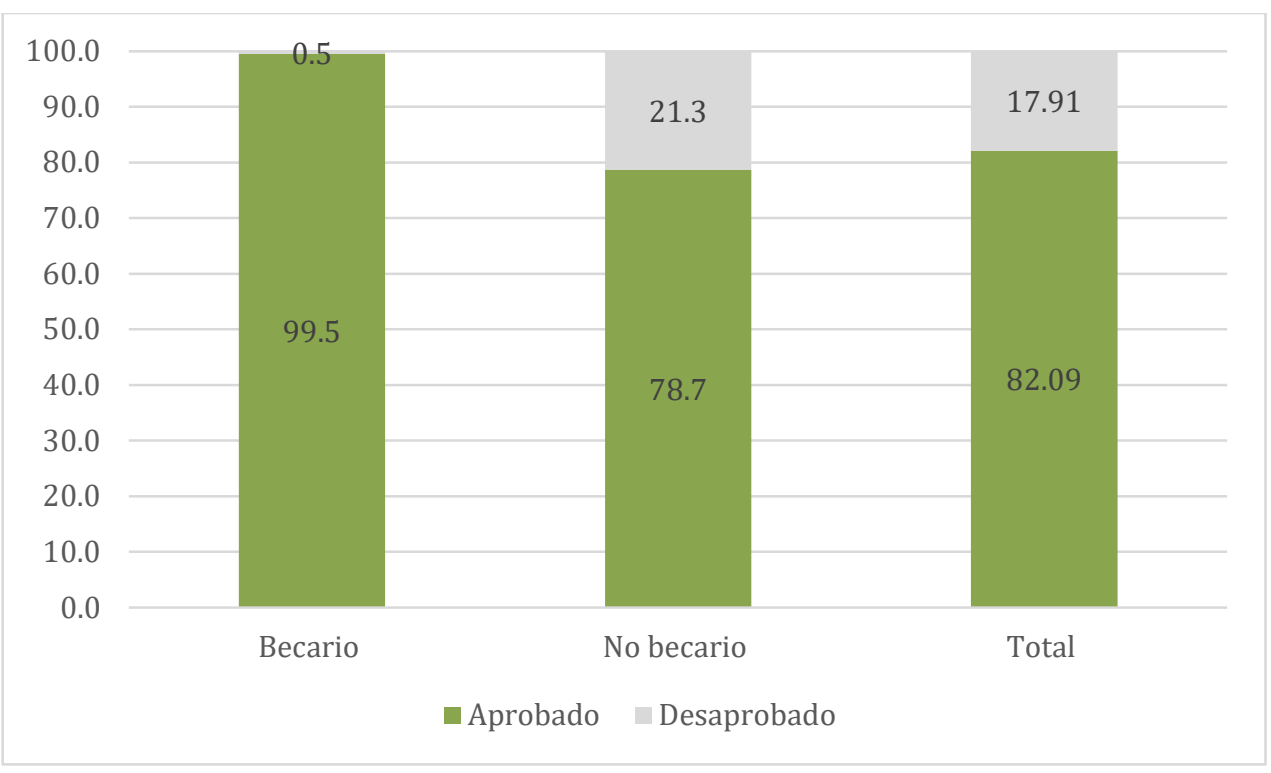

Figura 1. Porcentaje de aprobación de las materias por condición

Asimismo, los resultados descriptivos muestran que el valor de la media de los becarios $(M=14.8)$ se encuentra por encima de la media general $(M=12.4)$, y además es cuantitativamente superior a la media de los estudiantes no becarios $(M=11.9)$. Adicionalmente se puede observar una baja dispersión en el grupo de becarios ( $D E=1.4$ ), lo cual indica que los calificaciones son similares, mientras en el grupo de estudiantes no becarios encontramos una dispersión más elevada $(D E=4.25)$, lo cual revela que las calificaciones de este grupo de estudiantes no son parecidas.

Tabla IV. Análisis descriptivo del rendimiento académico de becarios y no becarios

\begin{tabular}{rrrr}
\hline & General & Becario & No becario \\
\hline $\mathrm{N}$ & 1262 & 204 & 1058 \\
Media & 12.3637 & 14.7959 & 11.8947 \\
Mediana & 13.4500 & 14.7400 & 13.0800 \\
Varianza & 16.634 & 1.965 & 18.105 \\
Desviación Estandar & 4.07843 & 1.40169 & 4.25496 \\
Mínimo & .00 & 9.06 & .00 \\
Máximo & 18.71 & 18.32 & 18.71 \\
\hline
\end{tabular}

\subsection{Análisis comparativo}

Para el análisis comparativo de las medias de calificaciones entre estudiantes becarios y no becarios, la comprobación se hizo a través del estadístico $t$ de Student, el cual se ejecutó con intervalo de confianza del 95\%. El resultado arrojó que el promedio de calificaciones del grupo de estudiantes becarios (14.8 \pm 1.4 puntos) fue significativamente mayor que del grupo de estudiantes no becarios (11.9 \pm 4.25 puntos).

Tabla V. Prueba $t$ de Student para muestras independientes con varianzas heterogéneas

\begin{tabular}{lcccccc}
\hline Valor $t$ & $\begin{array}{c}\text { Grados } \\
\text { de libertad }\end{array}$ & $\begin{array}{c}\text { Sig. } \\
\text { (bilateral) }\end{array}$ & $\begin{array}{c}\text { Diferencia } \\
\text { de medias }\end{array}$ & $\begin{array}{c}\text { Error típ. de } \\
\text { la diferencia }\end{array}$ & \multicolumn{2}{c}{$\begin{array}{c}\text { 95\% Intervalo de confianza } \\
\text { para la diferencia }\end{array}$} \\
\cline { 5 - 6 } & & & & Inferior & Superior \\
\hline 14.726 & 367.347 & .000 & 3.47224 & .23578 & 3.00859 & 3.93590 \\
\hline
\end{tabular}

Acorde con el planteamiento $t$ de Student para varianzas heterogéneas, se constató que el valor

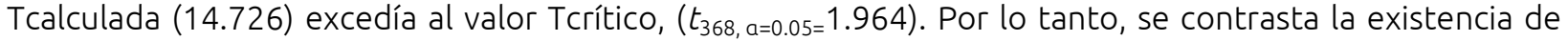


diferencias de medias en ambos grupos.

Procediendo a la estimación del tamaño del efecto mediante la $d$ de Cohen, en la tabla VI se observa que en promedio los becarios tienen una puntuación más alta en rendimiento académico (RA) a diferencia de los no becarios $\left(\mathrm{RA}_{\text {becarios }}=14.7959\right.$; $\mathrm{RA}_{n o}$ becarios $=1.40169$ ), siendo la magnitud de la diferencia grande $(d=0.915)$.

Tabla VI. Magnitud del efecto en relación al rendimiento académico en estudiantes becarios y no becarios

\begin{tabular}{rccccc}
\hline & \multicolumn{2}{c}{ Becarios } & \multicolumn{2}{c}{ No becarios } \\
\hline Variable & Media & desviación & Media & desviación & d de Cohen \\
\hline Rendimiento Académico & 14.7959 & 1.40169 & 11.8947 & 4.25496 & 0.9155362 \\
\hline
\end{tabular}

\section{Discusión y conclusiones}

El programa Beca 18 está focalizado en la población de adolescentes y jóvenes provenientes de sectores con desventajas socioeconómicas, a quienes se les brinda subvención económica y acompañamiento tutorial en el proceso de formación en una carrera profesional. El estudio analizó si la condición de becario tiene un efecto en el rendimiento académico de beneficiarios del programa; los resultados evidenciaron que los estudiantes becarios y no becarios alcanzan en general un alto porcentaje de aprobación de las asignaturas (82\%); no obstante, el análisis estadístico y de magnitud de efecto constata que el promedio de calificaciones alcanzado por los becarios $(M=14.8)$ es superior al de estudiantes no becarios $(M=11.8)$.

Los hallazgos de la investigación reafirman los resultados y conclusiones de estudios desarrollados por Navarro, (2015), Mediavilla (2013) y Estavillo et al. (2011). Por otra parte, reporta aspectos contrarios a los señalador por Catalán y Santelices (2015), Gómez (2014), Martínez y Martínez (2013), Berlanga et al. (2013) y Martínez (2012).

Con relación a los resultados presentados por Navarro (2015), si bien existe similitud en las conclusiones que desprende del análisis de datos, cabe señalar que el estudio se remite a una muestra pequeña de estudiantes ( $\mathrm{N}=25)$; además, no se efectuó un procesamiento estadístico de mayor rigurosidad, constituyendo los resultados de la presente investigación, el antecedente que aborda en el contexto peruano la variable del rendimiento académico en una muestra mayor de beneficiarios del programa Beca 18. De igual forma, se evidencian conclusiones similares a la de Mediavilla (2013); sin embargo, en el referido estudio el efecto diferencial positivo destaca al grupo de mujeres, variable que no se consideró. Asimismo, el estudio coincide con lo que afirman Estavillo et al. (2011), quienes siguieron un diseño metodológico similar a Mediavilla (2013), pero la muestra con la cual se trabajó no corresponde exclusivamente a estudiantes becados provenientes de sectores con desventajas socioeconómicas.

Por otro lado, se identificaron resultados contrarios a lo señalado por Catalán y Santelices (2015), los cuales pueden explicarse por las diferencias en relación con la cantidad de la muestra y el tiempo de recolección de datos. Asimismo, se afirma lo opuesto a lo mencionado por Gómez (2014) y Martínez y Martínez (2013), quienes centran la interpretación de sus resultados a nivel de la significancia estadística, no exponiendo sus hallazgos en una dimensión práctica. Se evidencia también lo contrario a lo encontrado por Berlanga et al. (2013), sin embargo, a diferencia de ellos, el presente estudio no consideró aspectos motivacionales, y queda como tarea pendiente comparar si los criterios de renovación de Beca 18 influyen en el rendimiento académico de los beneficiarios de este programa, a diferencia de otros estudiantes becados. Los resultados obtenidos contradicen también los obtenidos por Martínez (2012), los cuales están centrados en una población escolar y en resaltar la relación de la dotación de beca con la reducción del ausentismo escolar, variable no incluida en este estudio.

Algunas de las investigaciones citadas muestran resultados que relacionan positivamente la condición de becario y desempeño académico, así como estudios que señalan lo contrario. La particularidad de cada una de las evidencias que se discuten permite corroborar la explicación de la multiplicidad de factores asociados al rendimiento académico (Garbanzo, 2013; Garzón et al., 2010; Ruiz et al., 2007). En el caso de 
los beneficiarios de Beca 18, la asignación de la beca considera la presencia de un buen rendimiento en los últimos años de la educación básica (>=15 puntos en una escala vigesimal), por tanto, es posible que el factor de aptitud esté influyendo en el desempeño mostrado en los estudios universitarios, tal como lo señalan Miñano et al. (2008).

El estudio no consideró variables motivacionales, las cuales están relacionadas con el rendimiento académico (Donolo et al., 2011; Gutiérrez y López, 2012), pendiente para futuras investigaciones. Resulta pertinente destacar el carácter dual del programa Beca 18, que como lo señala Cuellar (2011) hace innovadora a esta propuesta, no remitiéndose exclusivamente a una subvención de corte económico, sino al acompañamiento tutorial. Con base en esta afirmación, el componente de tutoría y acompañamiento del programa tiene un valor explicativo en el rendimiento académico de los estudiantes beneficiarios, coincidiendo con lo reportado por Navarro (2015) y Rivera (2012).

Es importante visualizar la relación de variables socioculturales en el proceso educativo y sus resultados en la inclusión exitosa de estudiantes becarios, por lo cual los aspectos señalados por Chiroleu (2009) requieren se considerados en estudios a futuro. Asimismo, amerita desarrollar investigaciones desde la perspectiva de los becarios, como el efectuado por Rivera (2012), lo que conllevaría a entender de forma integral aspectos inmersos en su rendimiento académico, así como trazar nuevas líneas de investigación.

Cabe destacar que la UPN no aplica una encuesta censal que permita conocer las características socioeconómicas de los no becarios participantes del estudio, por ello es necesario, en futuras investigaciones, delimitar la variable socioeconómica de muestras de becarios y no becarios, pudiendo conocer su implicancia en el rendimiento académico.

En función a los resultados obtenidos, su análisis y discusión se concluye que el programa Beca 18 tiene un efecto positivo grande en el rendimiento académico de estudiantes becarios, expresado en el alto porcentaje de aprobación de materias, y en un mayor promedio de calificaciones en comparación con estudiantes no becarios.

Por último, es importante señalar que es necesario replicar estudios de este tipo en otras universidades que acogen a estudiantes becarios, puesto que políticas públicas como Beca 18 demandan inversión de capital humano y económico por parte del Estado, de las cuales muchas veces se desconoce su efectividad.

\section{Referencias}

Berlanga, V., Figuera, P. y Pons-Farnals, E. (2013). Incidencia de la beca salario: impacto, perfil y rendimiento de los estudiantes [Impact of the "salary scholarship" impact profile and student achievement]. Revista Electrónica de Investigación y Evaluación Educativa, 19(2).

doi http://dx.doi.org/10.7203/relieve.19.2.2654

Bolívar, A. (2005). Equidad educativa y teorías de la justicia. Revista Iberoamericana sobre Calidad, Eficacia y Cambio en Educación, 3(2), 42-69.

Catalán, X. y Santelices, M. V. (2015). Becas y rendimiento académico en la universidad: el caso de la Pontificia Universidad Católica de Chile. Revista Iberoamericana de Evaluación Educativa, 8(1), 63-80.

Chiroleu, A. (2009). Políticas públicas de inclusión en la Educación Superior: los casos de Argentina y Brasil. Revista Pro-Posições, 20(2), 141-166.

Cuellar, D. (2011). El valor de las becas en la Educación Superior. Revista Mexicana de Investigación Educativa, 16(51), 1299-1304. 
Edel, R. (2003). El rendimiento académico: concepto, investigación y desarrollo. Revista Electrónica Iberoamericana sobre Calidad, Eficacia y Cambios en la Educación, 1(2), 1-15.

El Comercio (23 de enero de 2012). Todo sobre el programa de becas para universidades Beca 18. Recuperado de http://archivo.elcomercio.pe/sociedad/lima/todo-sobre-programa-becas-universidadesbeca-18-noticia-1364761

Estavillo, K., Peralta, N. y Torres, L. (2011). Incidencia de la beca del fondo de solidaridad en el avance, escolaridad y egreso de los estudiantes de la Universidad de la República que ingresan en el año 2002. (Monografía de grado). Universidad de la República, Uruguay.

Garbanzo, G. (2013). Factores asociados al rendimiento académico en estudiantes universitarios desde el nivel socioeconómico: Un estudio en la Universidad de Costa Rica. Revista Electrónica Educare, 17(3), 5787. Recuperado de http://www.revistas.una.ac.cr/index.php/EDUCARE/article/view/5258/5448

Garzón, R., Rojas, M. O., del Riesgo, L., Pinzón, M. y Salamanca, A. L. (2010). Factores que pueden influir en el rendimiento académico de estudiantes de Bioquímica que ingresan en el programa de Medicina de la Universidad del Rosario-Colombia. Educación Médica, 13(2), 85-96.

Gómez, F. J. (2014). El impacto de las becas escolares en el rendimiento académico de los alumnos de licenciatura de la UANL. Daena: International Journal of Good Conscience, 9(2), 1-11. Recuperado de http://www.spentamexico.org/v9-n2/A1.9(2)1-11.pdf

González, A., Donolo, D., Rinaudo, C. y Paoloni, V. (2011). Relaciones entre motivación, emoción y rendimiento académico en universitarios. Estudios de Psicología, 32(2), 257-270.

doi:10.1174/021093911795978207

Gutiérrez, M. y López, E. (2012). Motivación, comportamiento de los alumnos y rendimiento académico. Infancia y Aprendizaje, 35(1), 61-72. doi:10.1174/021037012798977421

Ledesma, R., Macbeth, G. y Cortada, N. (2008). Tamaño del efecto: revisión teórica y aplicaciones con el sistema estadístico ViSta. Revista Latinoamericana de Psicología, 40(3), 425-439.

Martínez, E. y Martínez, R. (2013). Análisis del efecto de las becas económicas en el rendimiento escolar en una institución de nivel superior medio. Investigación y Ciencia, 59, 41-47.

Martínez, O. A. (2012). Efectos de las becas educativas del programa Oportunidades sobre la asistencia escolar. El caso de la zona urbana del noreste de México. Desarrollo y Sociedad, 69, 99-131.

Mediavilla, M. (2013). Heterogeneidad en el impacto de la política de becas en la escolaridad secundaria postobligatoria en España: un análisis por subgrupos poblacionales. Estudios de Economía, 40(1), 97-120.

Ministerio de Educación (2014). Memoria institucional del programa de becas y crédito educativo del Ministerio de Educación. Perú: Autor. Recuperado de

http://www.pronabec.gob.pe/inicio/publicaciones/documentos/memoria 2014.pdf

Miñano, P., Cantero, M. y Castejón, J. (2008). Predicción del rendimiento escolar de los alumnos a partir de las aptitudes, el autoconcepto académico y las atribuciones causales. Revista Horizontes Educacionales, 13(2), 11-23.

Montero, I. y León, O. G. (2007). A guide for naming research studies in Psychology. International Journal of Clinical and Health Psychology, 7(3), 847-862.

Navarro, O. (2015). Rendimiento académico en estudiantes del Programa Nacional Beca 18 en la Universidad Continental. Apuntes en Ciencias Sociales, 5(1), 27-31. doi:10.18259/acs.2015005 
Rivera, G. (2012). Sistematización de los programas de becas integrales "RP. Jorge Dintilhac SS.CC." y "Fe y Alegría" de la Pontificia Universidad Católica del Perú. (Tesis de maestría). Pontificia Universidad Católica del Perú. Recuperado de http://tesis.pucp.edu.pe/repositorio/handle/123456789/4467

Ruiz, E., Ruiz, G. A. y Odstrcil, M. (2007). Metodología para realizar el seguimiento académico de alumnos universitarios. Revista Iberoamericana de Educación, 42(3), 2-9. Recuperado de http://rieoei.org/deloslectores/1590Ruiz.pdf 